\title{
THE PREDICTIVE ROLE OF THE HEALTH BELIEFS IN DIFFERENT FORMS OF ADAPTIVE BEHAVIOR RELATED TO THE COVID-19
}

This work was done within the research grant from the RA science committee №20TTSH-070. Khachatryan N. G., Grigoryan A. K. (Yerevan State University, Yerevan, Armenia) n_khachatryan@ysu.am,ani_grigoryan@ysu.am 乙tipluјшgर्üи uर्u. 05.08.2021 9pmpunuर्uir uर्रu. 14.08.2021

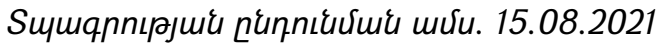

Health campaigns and educational programs have recently focused on forming people's beliefs. Many studies show that beliefs contribute to the development of preventive health behaviors.

The purpose of this study was to investigate the predetermining role of health beliefs about Covid-19 (i.e., health belief model) for adaptive behaviors related to the pandemic. We focused on preventive, hoarding, and helping behaviors as common reactions toward the pandemic. The study was based upon a sample of 237 participants $(64,1 \%$ woman, $M=29,75 ; S D=10,693$; Range=18-58). Data collection occurred between May and June 2020 through an online survey. The results of the hierarchic regression analysis showed that the preventive behavior was determined by sex, beliefs about the effectiveness of the preventive behavior, perception of barriers, perception of severity and fear of Covid-19. The economic status and beliefs of gaining prevention were significant predictors for hoarding behavior. The sex, fact of being infected, and the fear of Covid-19 predicted the helping behavior. None of the health beliefs was a predictor for this behavior. The results of the study provide an opportunity to understand the role of health beliefs about Covid-19 and other factors in the manifestation of different behaviors.

Keywords: Health belief model, preventive, hoarding and helping behaviors, fear of Covid-19.

DOI: https://doi.org/10.46991/SBMP/2022.5.1.063

2020p. Covid-19-h pnulynuरa unmqugntig unmpptp hulymqnnuरatip' mun-

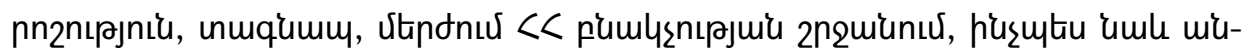

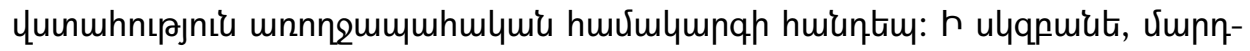

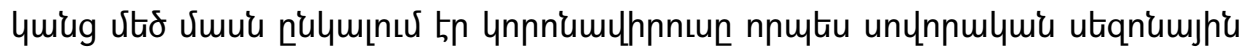

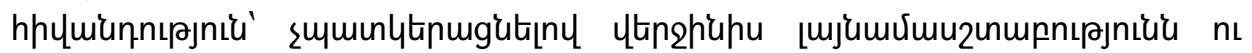

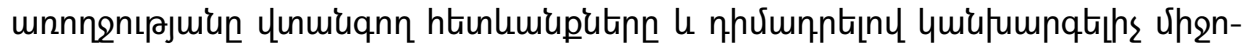




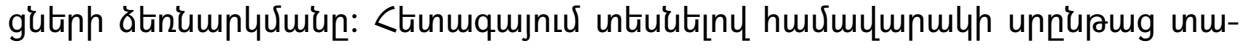

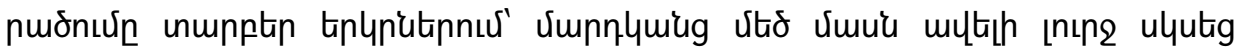

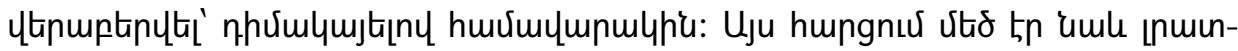

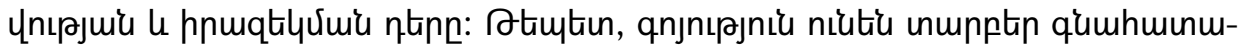

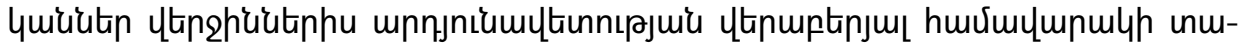

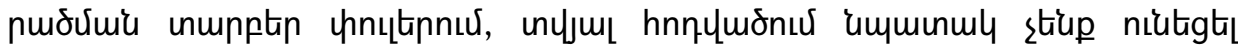

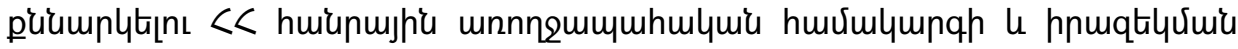

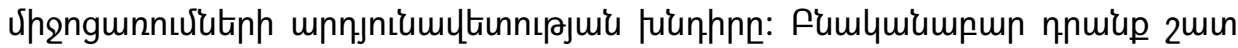

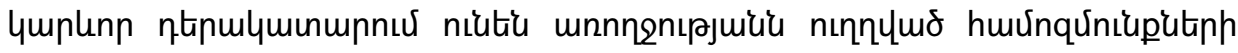

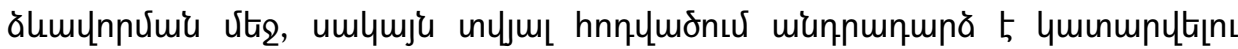

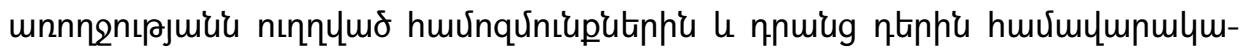

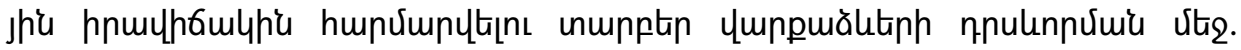

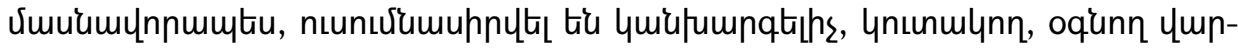

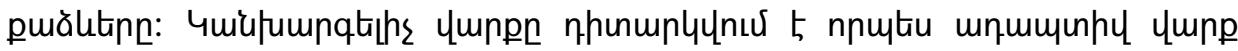

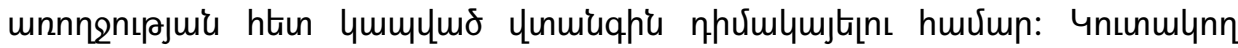

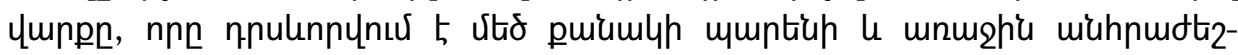

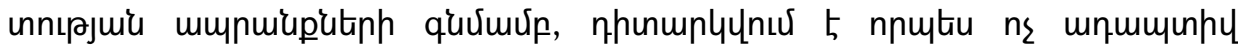

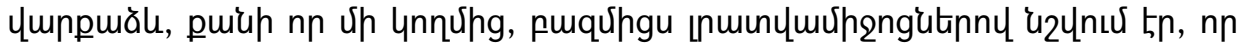

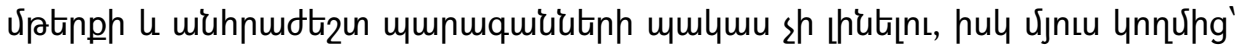

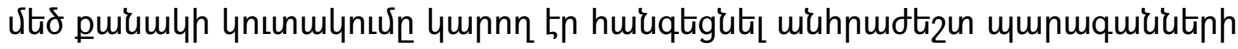

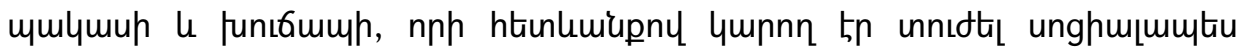

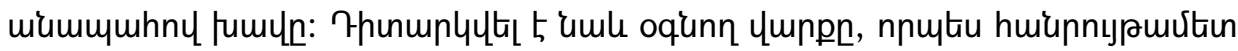

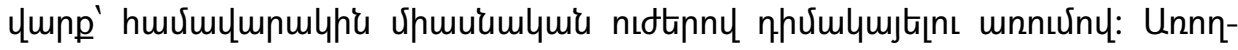

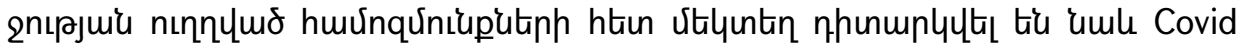

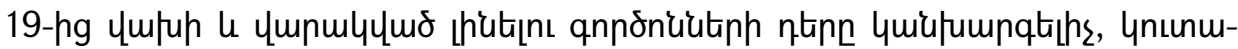
unn 4 oqunn ymppuăltiph huxump:

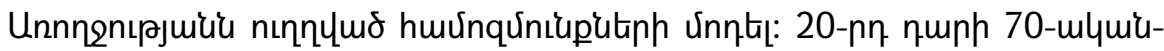

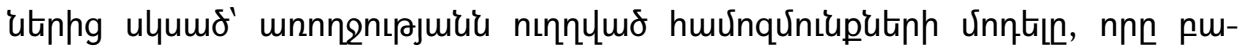

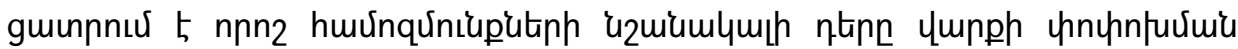

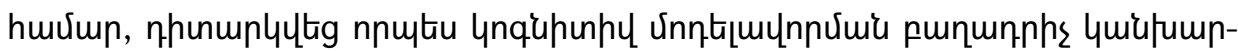

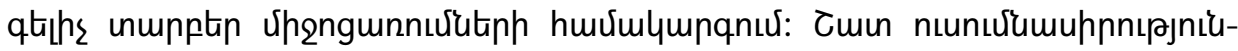

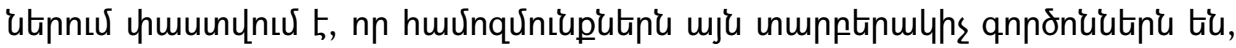

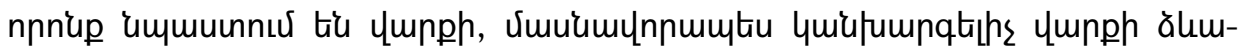

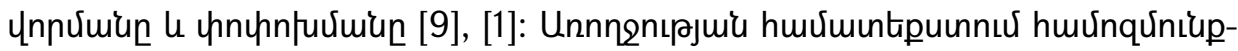

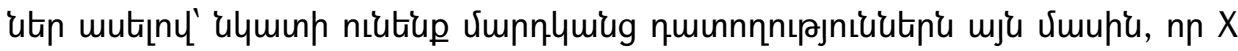

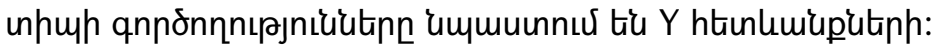

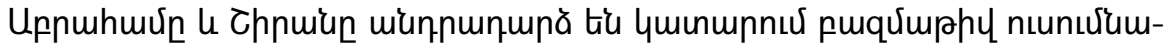

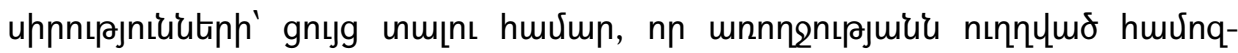

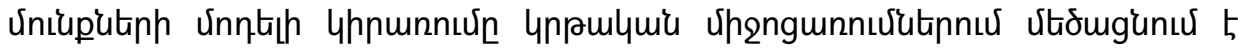

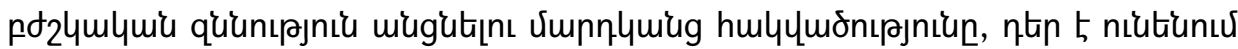




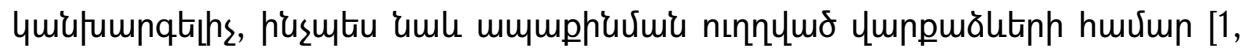
30-31] :

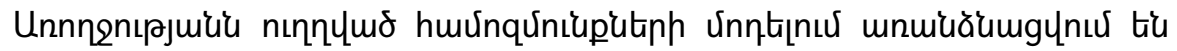
htinlumu punumphšutinn [4, 74].

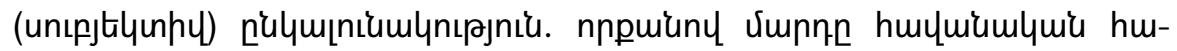

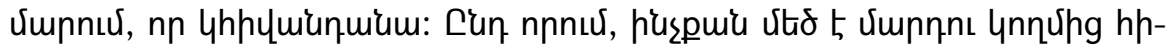

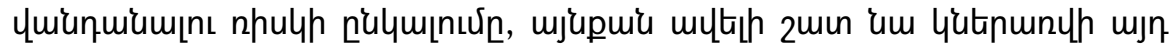
nhulp ulumqtigumiu:

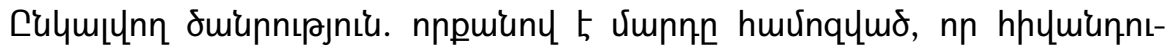

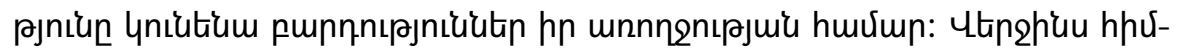

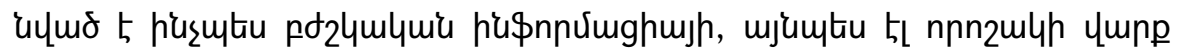

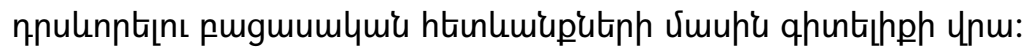

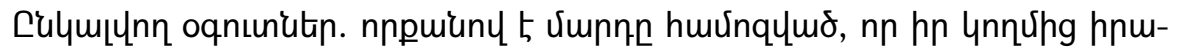

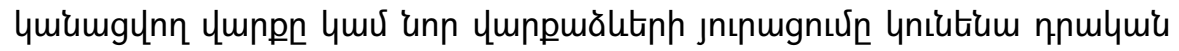

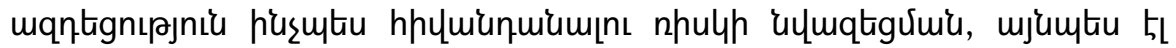

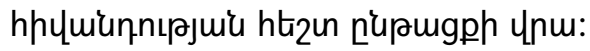

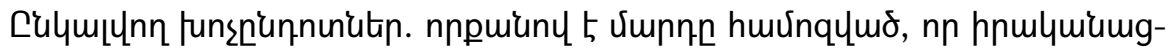

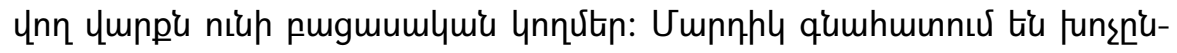

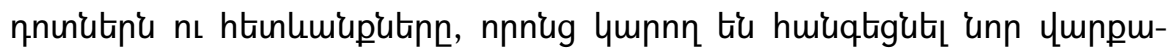

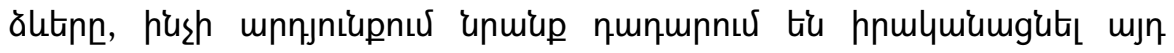
yurpn [5], [6], [7]:

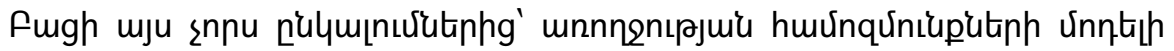

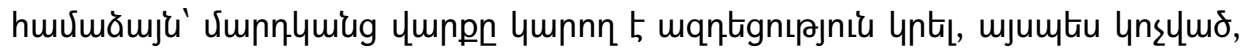

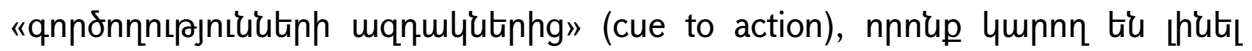

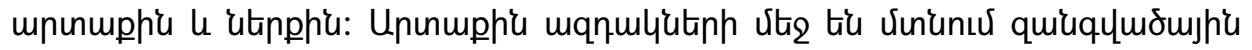

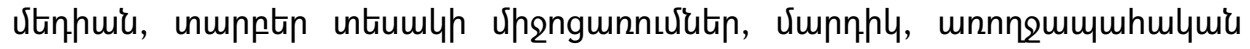

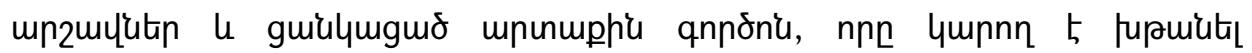

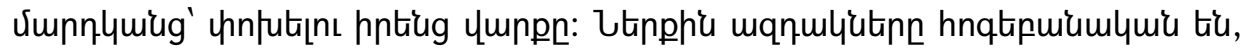

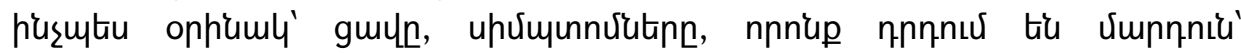
jnınugütı̇ıı unn ymppuălu [3, 34]:

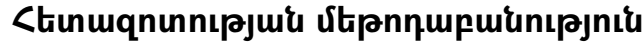

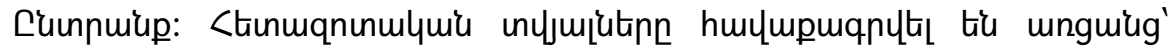

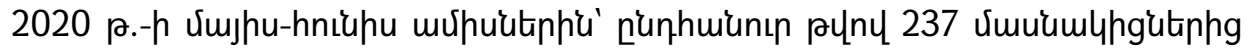
(64,1\% 4hu, $M=29.75 ; S D=10.693$; Range=18-58): Chunuqnunulquiu nuunnuiuph

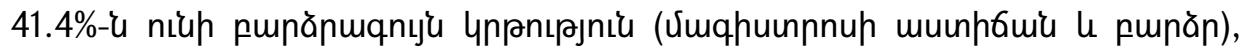

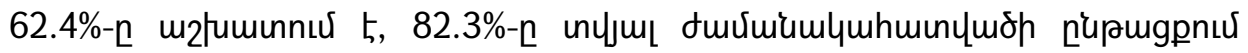

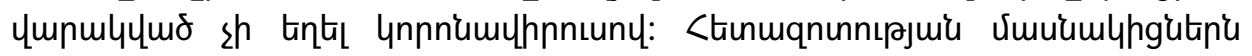

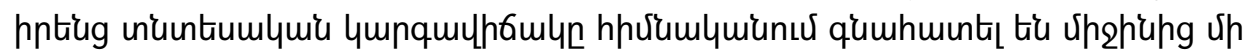

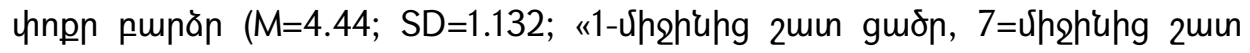

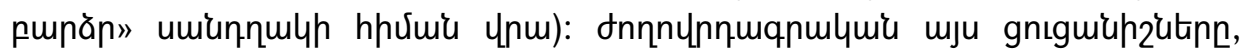




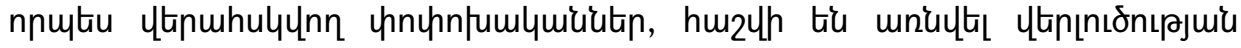
nupurgpnu:

\section{Chunuqnunnıpjuí qnndppulququ}

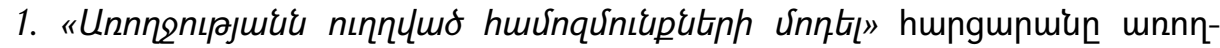

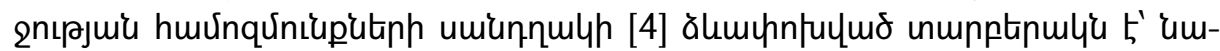

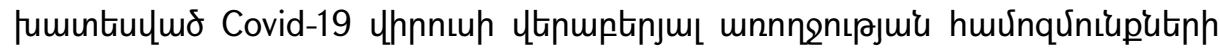

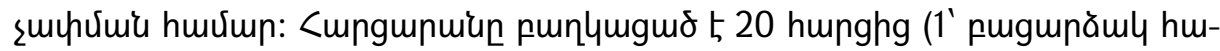

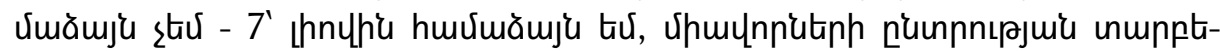

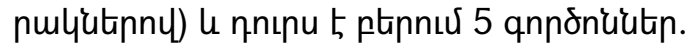

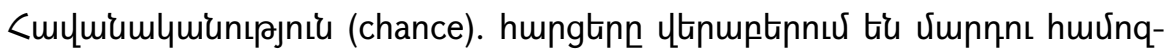

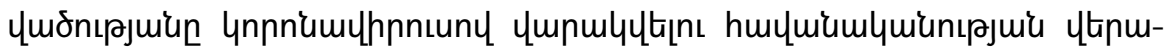

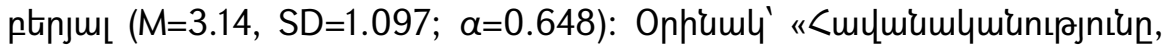

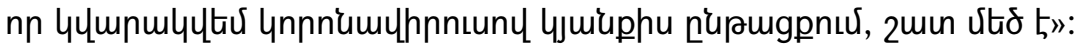

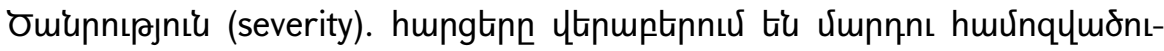

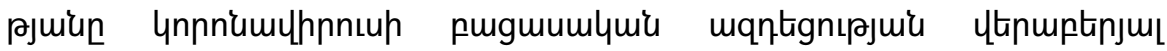

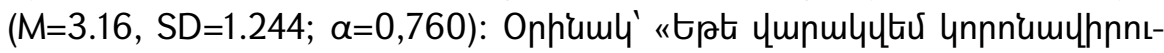

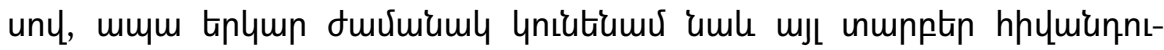
pjniưutitn»:

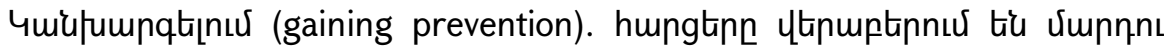

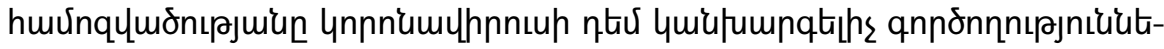

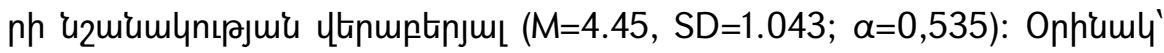

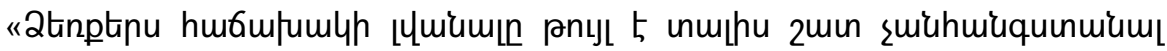

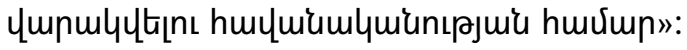

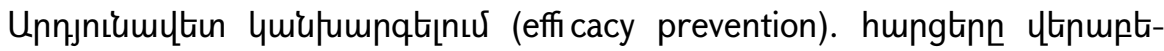

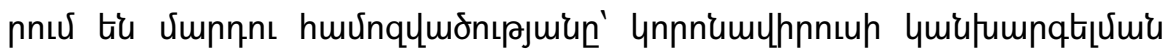

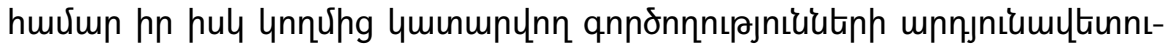

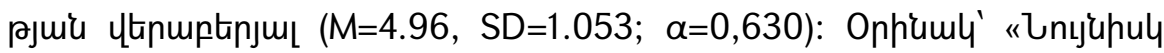

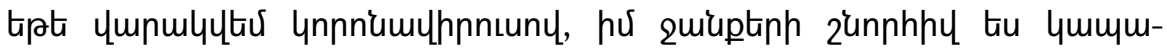
phuytuu»:

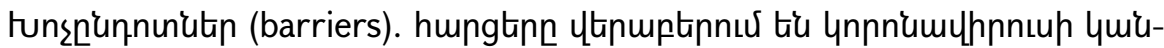

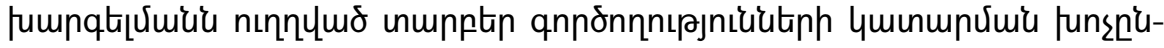

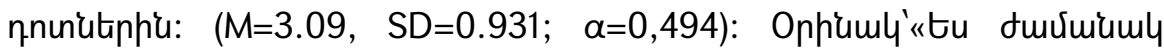

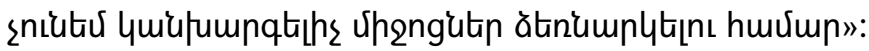

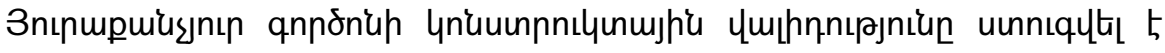

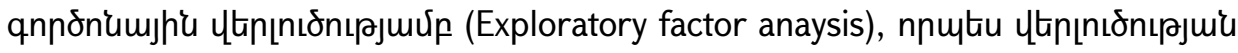

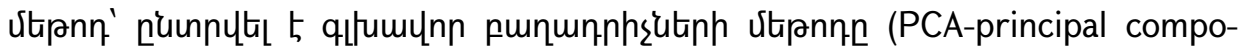

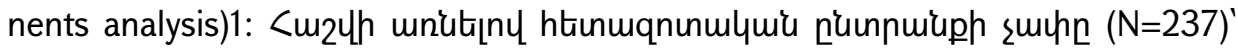

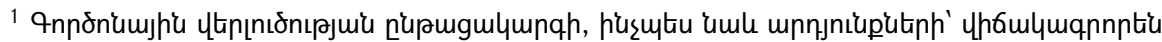

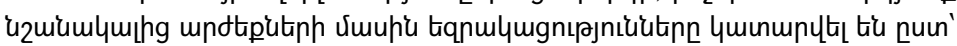




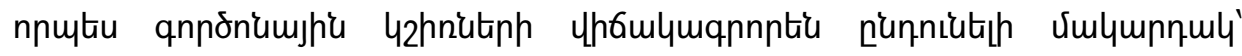

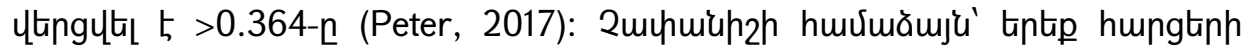

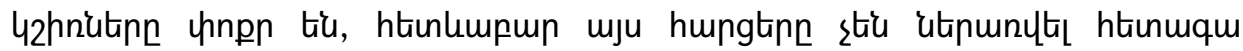

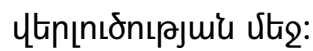

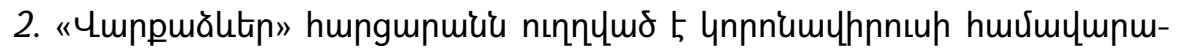

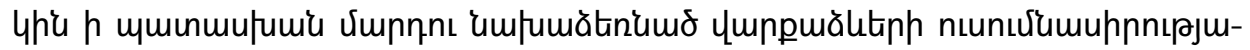

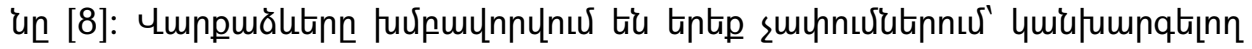

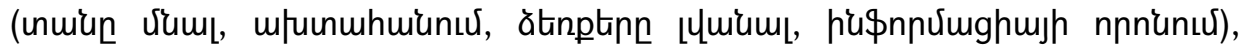

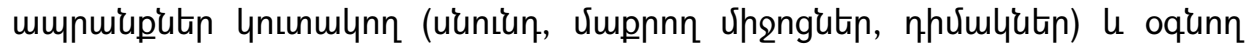

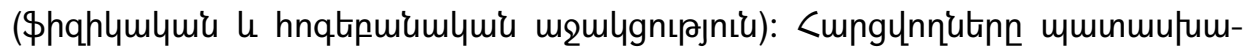

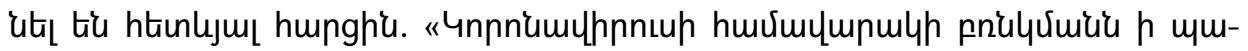

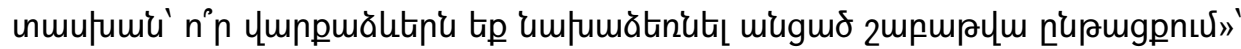

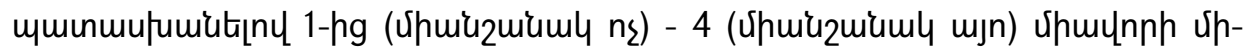

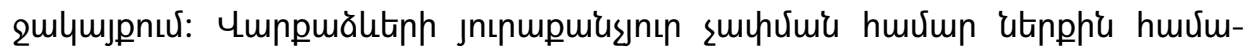

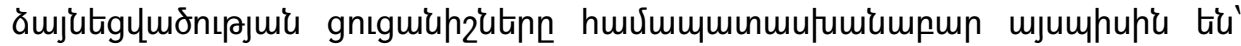
0.733; 0.713, 0.607 ( $M 1=2.91, S D 1=0.735 ; M 2=2.77, S D 2=0.811 ; M 3=2.09$,

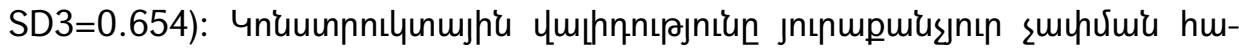

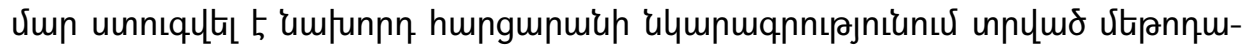

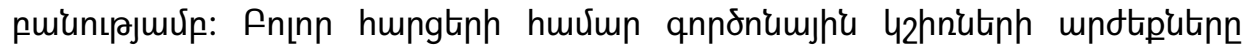

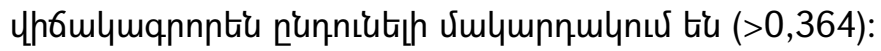

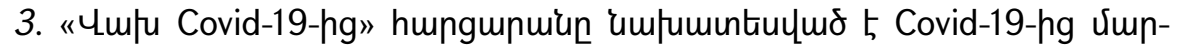

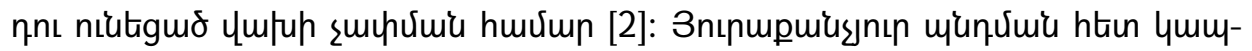

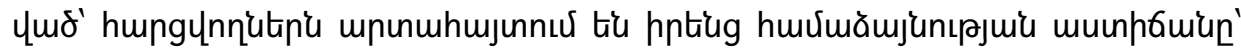

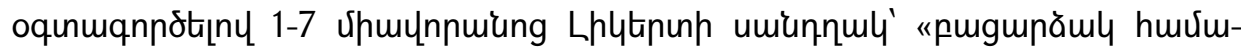

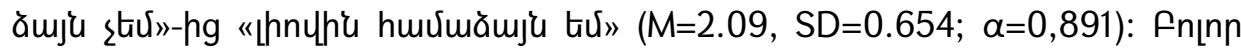

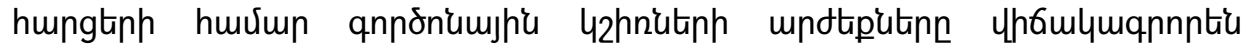

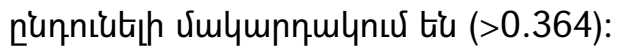

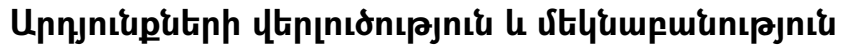

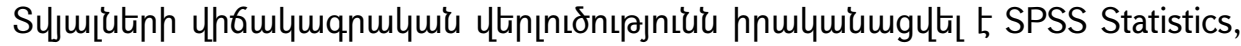

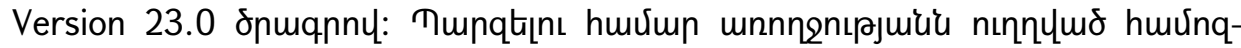

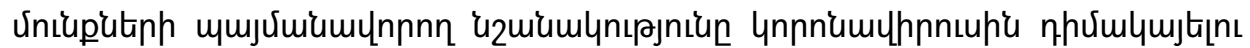

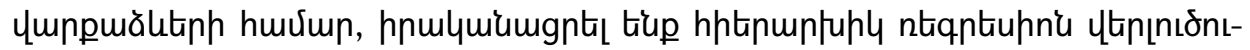

\footnotetext{
Peter S. (2017) Advice on Exploratory Factor Analysis// http://www.openaccess.bcu.ac.uk/6076/1/_staff_shares_storage\%20500mb_Library_ID112668_Stats\%20Advisory_Ne w\%20Statistics\%20Workshops_18ExploratoryFactorAnalysis_ExploratoryFactorAnalysis4.pdf

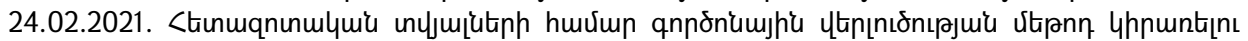

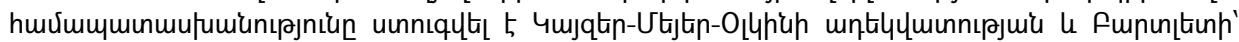

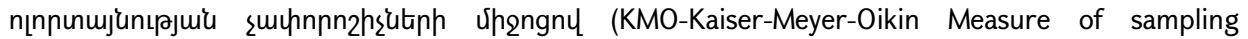

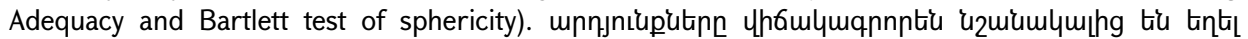

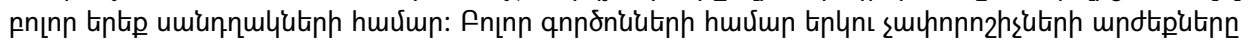

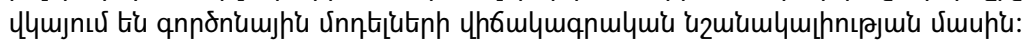




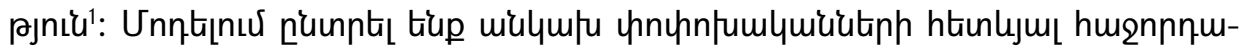

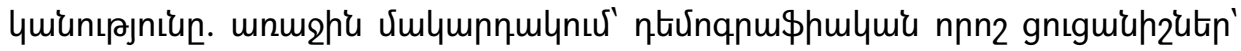

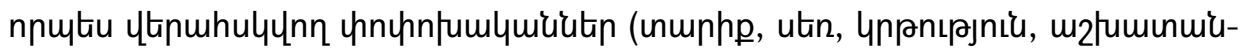

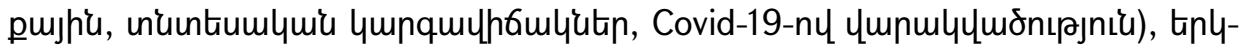

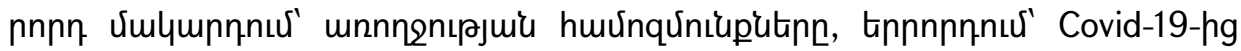

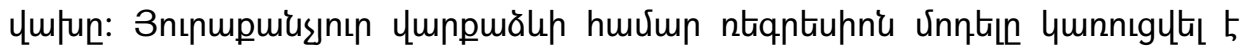

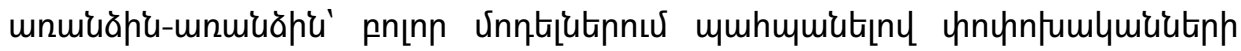

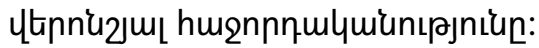

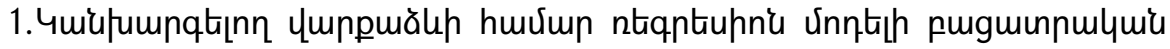

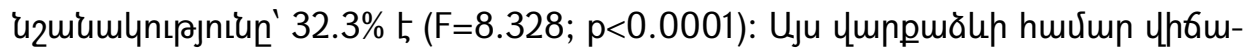

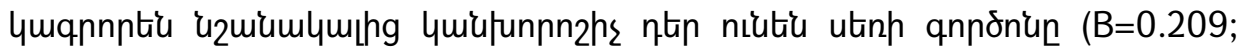

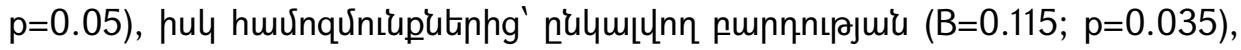

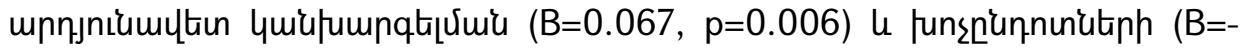

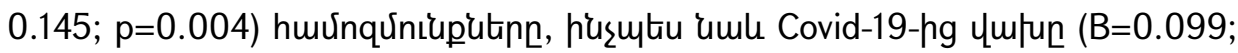

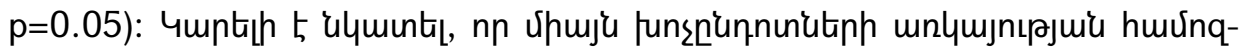

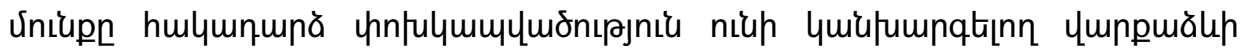

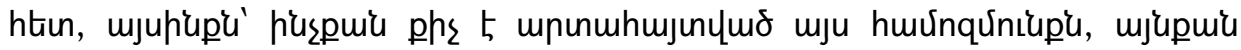

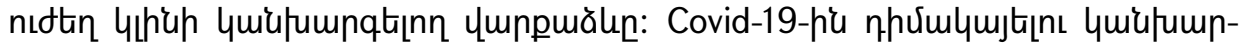

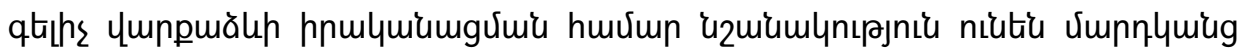

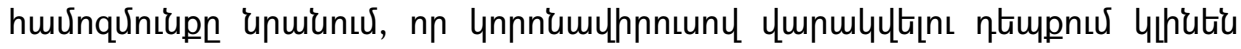

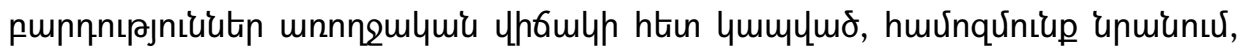

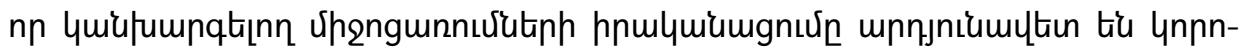

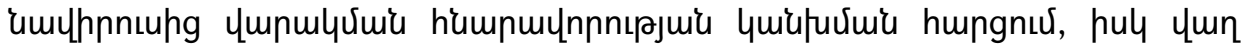

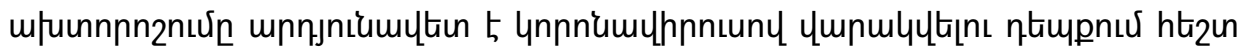

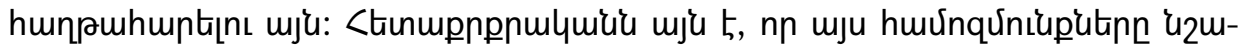

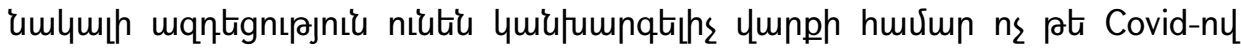

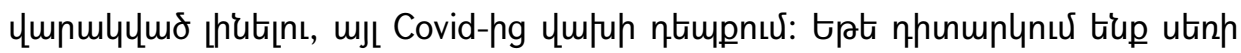

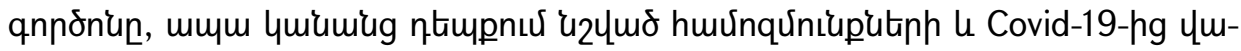

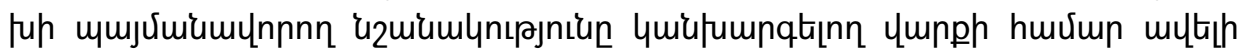
utid t:

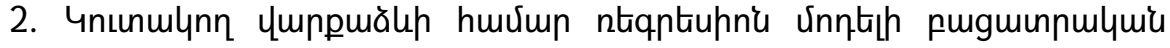

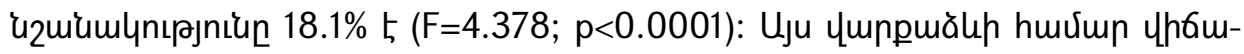

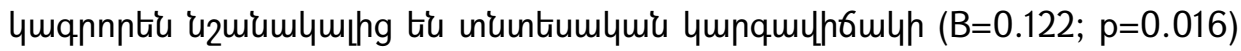

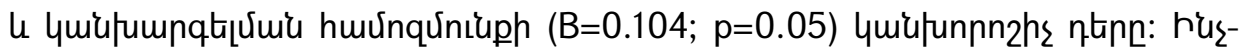

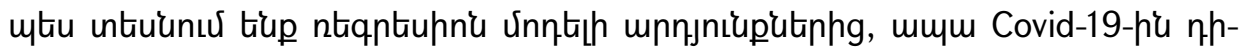

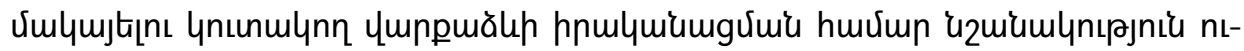

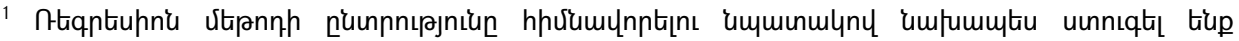

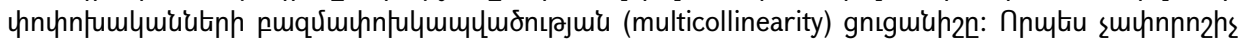

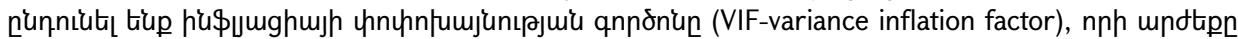

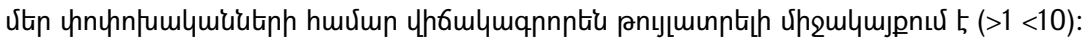




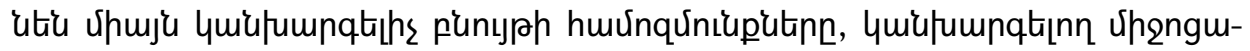

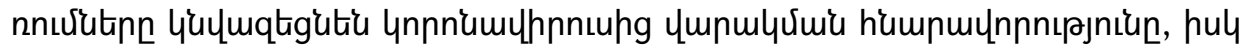

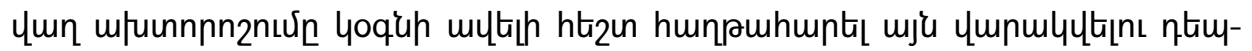

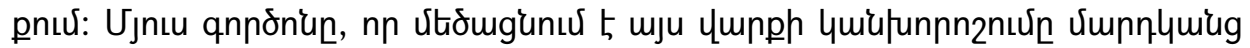

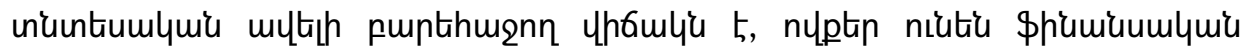

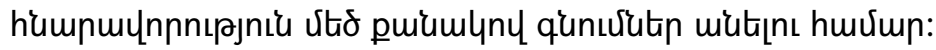

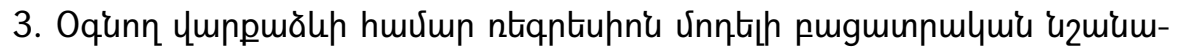

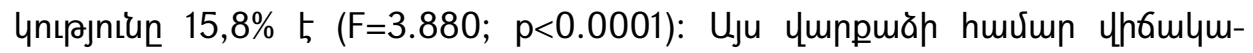

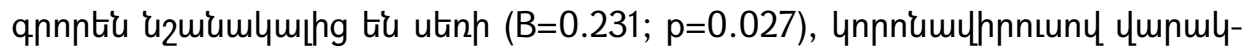

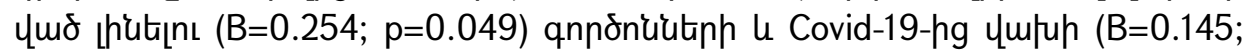

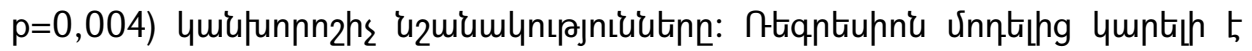

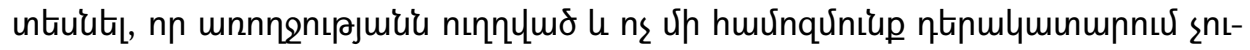

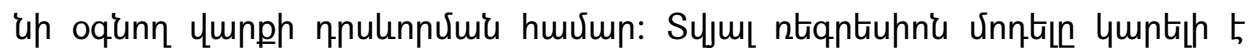

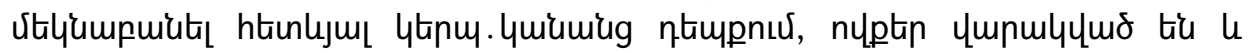

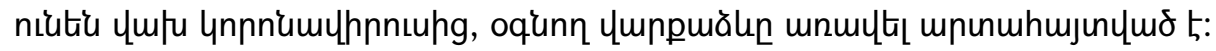

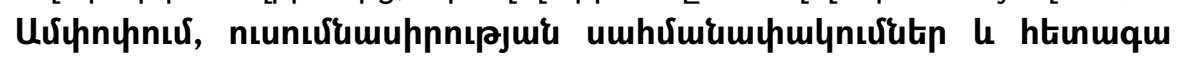

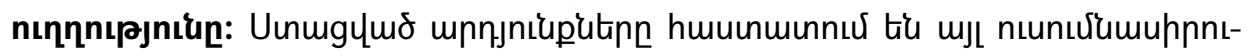

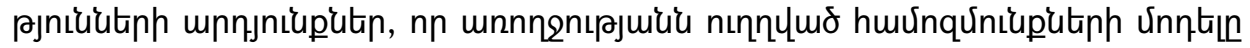

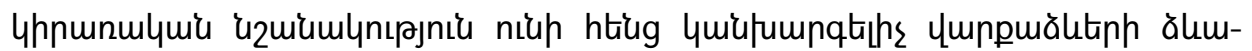

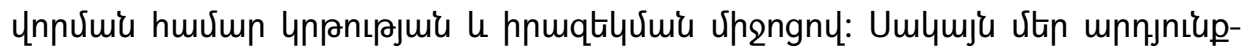

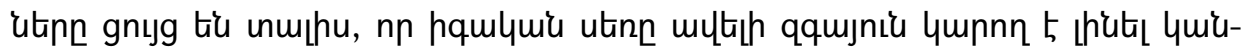

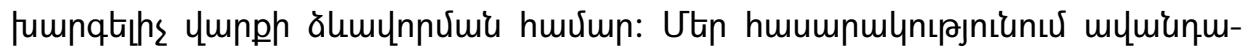

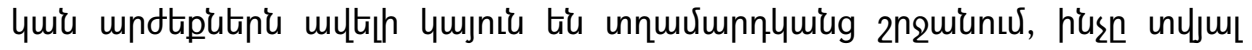

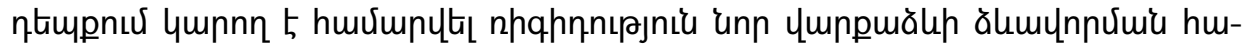

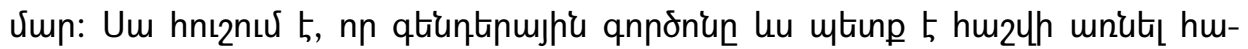

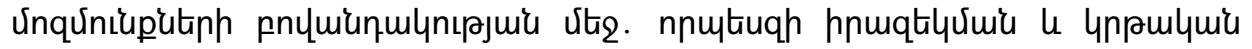

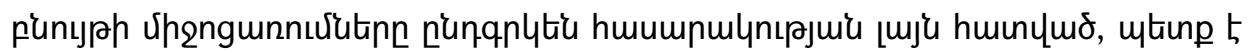

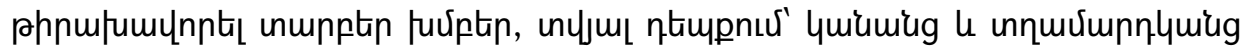

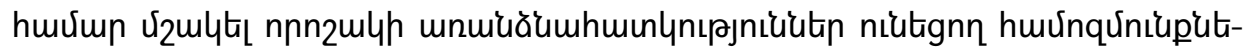

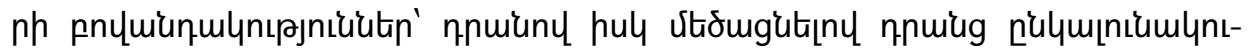
PJniun:

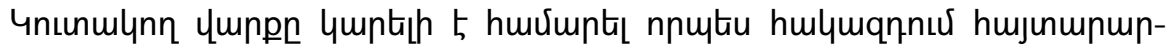

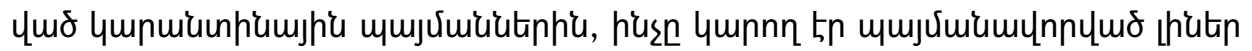

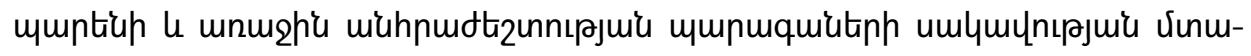

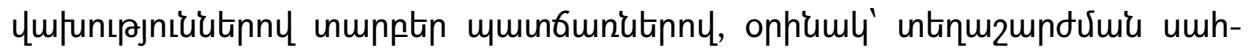

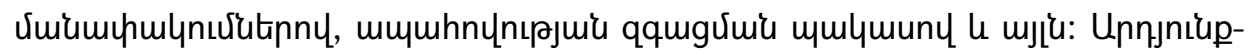

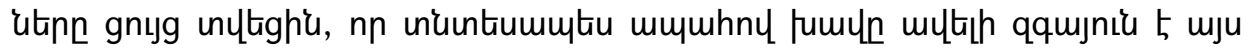
чunpuăle huiuntru:

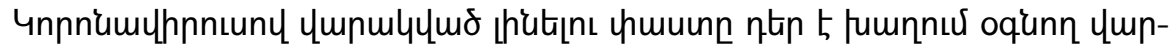

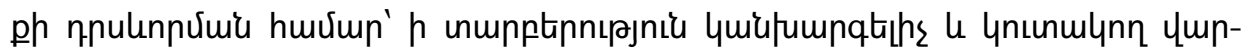




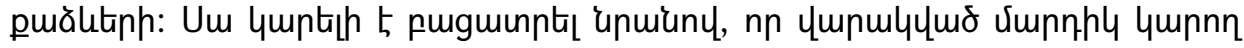

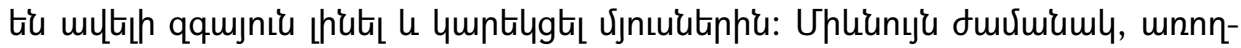

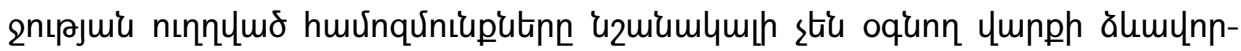

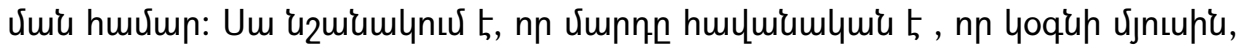

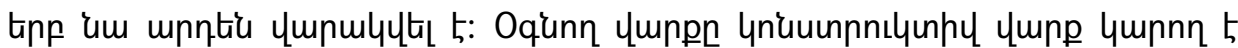

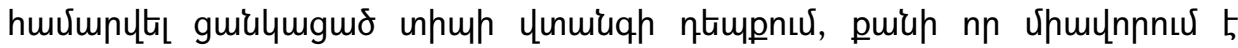

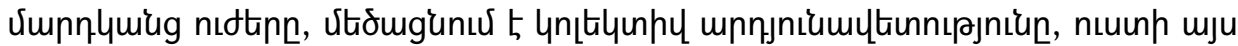

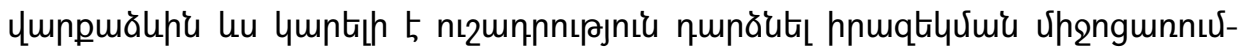
utinnıu:

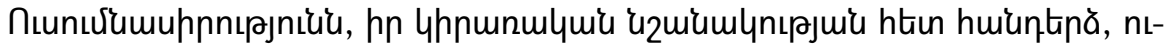

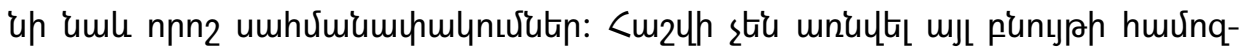

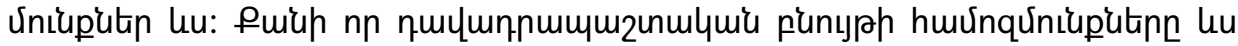

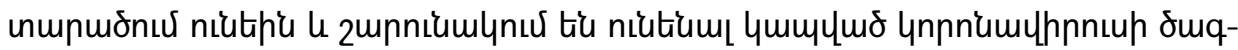

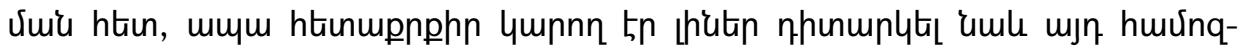

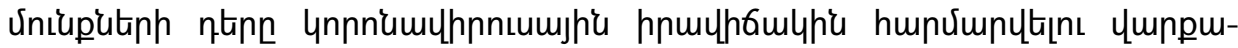

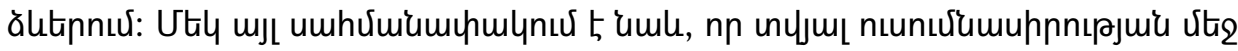

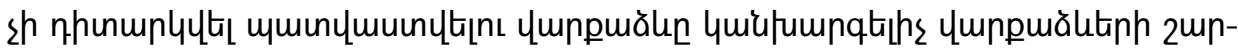

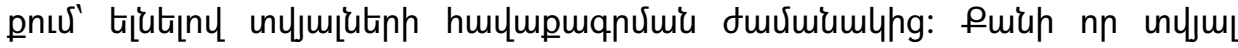

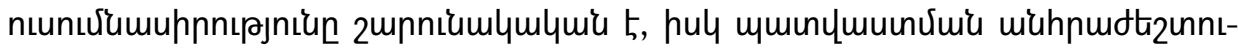

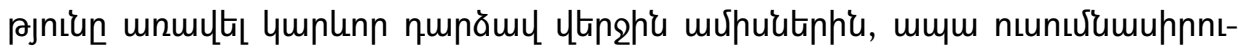

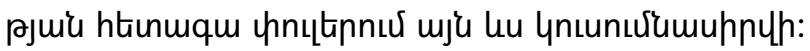

\section{9pulquintpjnıtu}

1. Abraham C., Sheeran P. The health belief model. In Predicting and changing health behaviour: Research and Practice with Social Cognition Models (ed. Conner M. \& Norman P.), $3^{\text {rd }}$ ed., Open University press, 2015, pp. 30-65.

2. Ahorsu DK, Lin CY, Imani V, Saffari M, Griffiths MD, Pakpour $\mathrm{AH}$. The Fear of COVID-19 Scale: Development and Initial Validation. Int J Ment Health Addict. 2020; pp. 1-9.

3. Alsulaiman S.A., Rentner T.L. The Health Belief Model and Preventive Measures: A Study of the Ministry of Health Campaign on Coronavirus in Saudi Arabia, Journal of International Crisis and Risk Communication Research, 1(1), 2018, pp. 27-56.

4. Champion, V.L. Instrument development for health belief model constructs, Advances in Nursing Science, 6, 1984, pp. pp.73-85.

5. Glanz, K., Rimer, B. K., \& Lewis, F. M. Health behavior and health education: Theory, research, and practice. San Francisco, CA: JosseyBass, 2002, 512p. 
6. Hayden, J. Introduction to health behavior theory (2nd ed.). Burlington, MA: Jones \& Bartlet, 2013.

7. Janz N. K., \& Becker, M. H. The health belief model: A decade later. Health Education \& Behavior, 11, 1984, pp.1-47.

8. Nowak B., Brzóska P., Piotrowski J., Sedikides C., ŻemojtelPiotrowska, M., Jonason, P. K. Adaptive and maladaptive behavior during the COVID-19 pandemic: The roles of Dark Triad traits, collective narcissism, and health beliefs. Personality and Individual Differences, 2020, 167.

9. Rozentock I. The Health Belief Model and Preventive Health Behavior. Health Education Monographs vol. 2, No. 4, 1975, pp.354386.

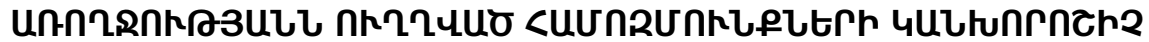

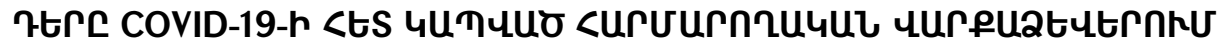

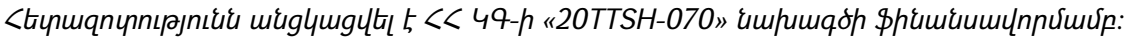

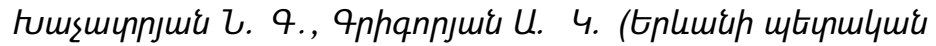

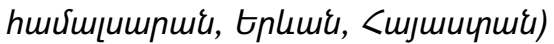

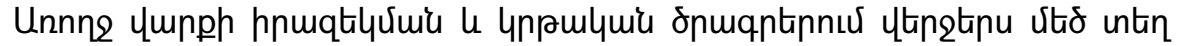

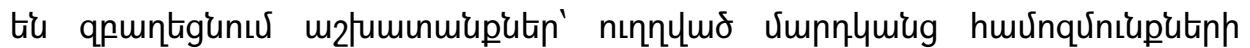

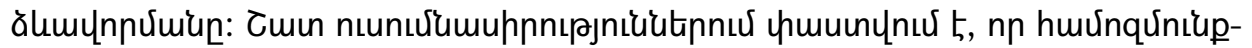

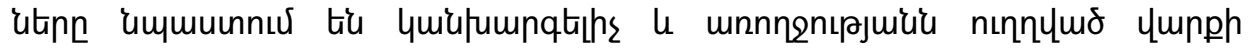

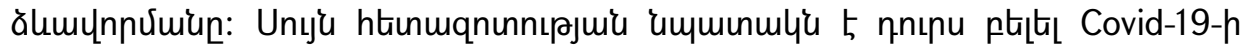

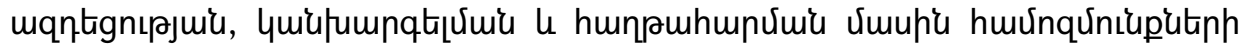

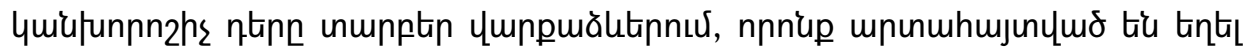

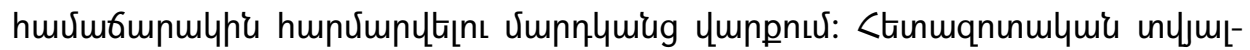

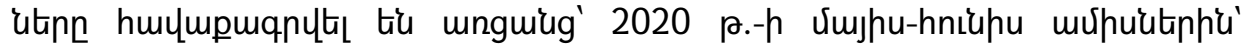
nunhwiunın puny 237 umu'umlyhgutinh (64,1\% uhu, $M=29,75 ; S D=10,693$;

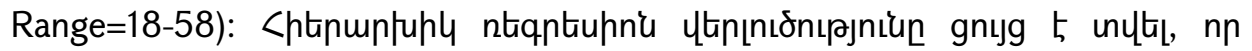

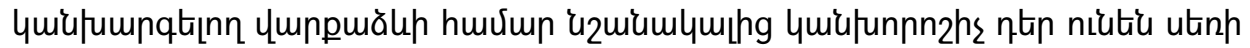

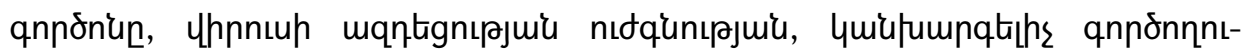

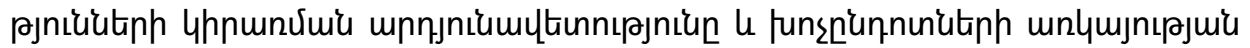

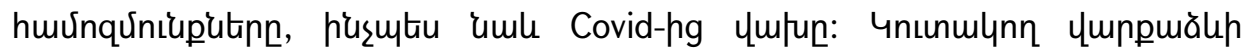

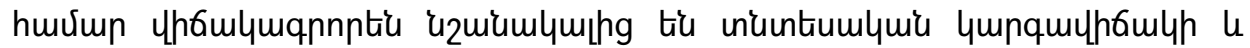

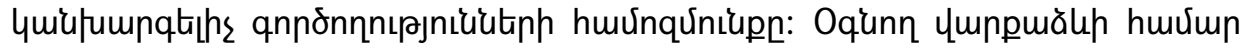

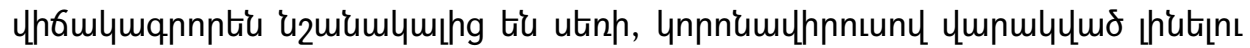

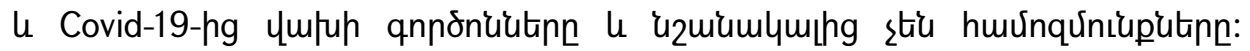

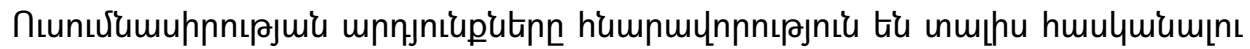




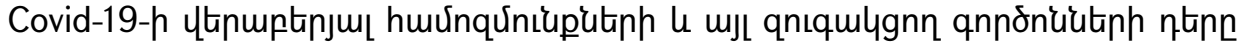

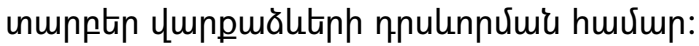

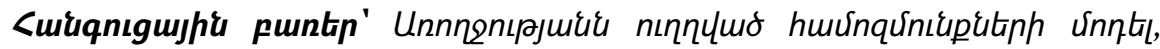

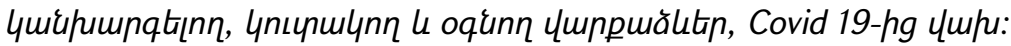

\section{ПРЕДИКАТИВАНАЯ РОЛЬ УБЕЖДЕНИЙ В ОТНОШЕНИИ ЗДОРОВЬЯ В РАЗЛИЧНЫХ ФОРМАХ АДАПТИВНОГО ПОВЕДЕНИЯ, СВЯЗАННЫХ С COVID-19}

Исследование выполнено в рамках научного гранта (20TTSH-070) KH PA: Хачатрян Н. Г., Григорян А. К. (Ереванский государственный университет, Ереван, Армения)

В образовательных и просветительских программах в последнее время большая роль отводится работам, направленным на формирование убеждений. Во многих исследованиях отмечается, что убеждения в отношении здоровья, способствуют поведению, направленного на здоровье.

Цель данного исследования - выявление предикативаной роли убеждений, связанных с влиянием, профилактикой и преоделением Covid-19 в разных формах поведения. Данные были собраны в онлайн режиме с мая по июнь 2020 года, в общем количестве 237 человек (64,1\% женщины, $\mathrm{M}=29,75 ; \mathrm{SD}=10,693$; Range=18-58). Иерархический регрессионный анализ показал, что для профилактического поведения оказались значимыми фрактор пола, страх к вирусу, убеждения, связанные с тяжестью последствий от вируса, с продуктивностью профилактических мер, а также с препятствиями для выполнения необходимых действий. Для накопительского поведения оказались значимыми фактор экономического благополучия, убеждение, связанное с важностью профилактических мер. Для помогающего поведения оказались значимыми фактор пола, а также страх к вирусу и факт заражения, и не оказались значимыми убеждения. Результаты исследования позволяют говорить о значимости убеждений и других сопряженных фракторов в обьяснении разных форм адаптивного поведения к Covid-19.

Ключевые слова: Модель убеждении в отношении здоровья, профилактическое, накопительское и помогающее поведения, страх к Covid-19. 